\title{
Existence of Solutions to a Viscous Thin Film Equation
}

\author{
Yue Qiu1,2, Bo Liang3 \\ ${ }^{1}$ Foundation Building, 765 Brownlow Hill, University of Liverpool, Liverpool, UK \\ ${ }^{2}$ Foundation Building, Xi'an Jiaotong-Liverpool University, Suzhou, China \\ ${ }^{3}$ School of Science, Dalian Jiaotong University, Dalian, China \\ Email: cnliangbo@163.com
}

How to cite this paper: Qiu, Y. and Liang, B. (2018) Existence of Solutions to a Viscous Thin Film Equation. Journal of Applied Mathematics and Physics, 6, 2119-2126. https://doi.org/10.4236/jamp.2018.610178

Received: September 24, 2018

Accepted: October 23, 2018

Published: October 26, 2018

Copyright (C) 2018 by authors and Scientific Research Publishing Inc. This work is licensed under the Creative Commons Attribution International License (CC BY 4.0).

http://creativecommons.org/licenses/by/4.0/

\begin{abstract}
A fourth-order degenerate parabolic equation with a viscous term: $\left\{\begin{array}{ll}u_{t}-\left(m(u) w_{x}\right)_{x}=0 & \text { in }(-1,1) \times(0, T), \\ w=-u_{x x}+v u_{t} & \text { in }(-1,1)) \times(0, T)\end{array}\right.$ is studied with the initial-boundary
\end{abstract} conditions $u_{x}=w_{x}=0$ on $\{-1,1\} \times(0, T), u(x, 0)=u_{0}(x)$ in $(-1,1)$. It can be taken as a thin film equation or a Cahn-Hilliard equation with a degenerate mobility. The entropy functional method is introduced to overcome the difficulties that arise from the degenerate mobility $m(u)$ and the viscosity term. The existence of nonnegative weak solution is obtained.

\section{Keywords}

Fourth-Order Degenerate Parabolic, Thin Film Equation, Cahn-Hilliard Equation, Entropy Functional

\section{Introduction}

In recent years, the research of nonlinear fourth-order degenerate parabolic equations has become an interesting topic. The typical examples include the Cahn-Hilliard equation and the thin film equation. The Cahn-Hilliard equation can describe the evolution of a conserved concentration field during phase separation. It (see [1]) has the form $u_{t}+\nabla \cdot\left(k \nabla\left(\varepsilon^{2} \Delta u+A^{\prime}(u)\right)\right)=0$ where the constants $k, A, \varepsilon^{2}$ denote the atomic mobility, the free energy, the parameter proportional to the interface energy respectively and $-\left(\varepsilon^{2} \Delta u+A^{\prime}(u)\right)$ is a kind of chemical potential. For the existence and the properties of solutions, Elliott, Zheng and Garcke (see [2] [3]) have studied this equation with a linear and a degenerate mobility respectively. Xu, Zhou, Liang and Zheng (see [4] [5] [6]) 
have applied the semi-discrete method to obtain the existence and stability results to this model with a gradient mobility.

The thin film equation can analyze the motion of a very thin layer of viscous incompressible fluids along an inclined plane or model the fluid flows such as draining of foams and the movement of contact lenses. The thin film equation belongs to a class of fourth order degenerate parabolic equations (see [7]) and the first mathematic result, the existence and nonnegativity of weak solutions, are given by Bernis and Friedman [8] to the equation $u_{t}+\left(u^{n} u_{x x x}\right)_{x}=0$. The thin film equation with a second-order diffusion term was studied by Bertozzi and Pugh [9]. Moreover, for a generalized thin-film equation with period boundary in multidimensional space, Boutat et al. [10] obtained its existence. For other results, the readers may refer to the papers [11] [12].

In this paper, we study the following initial and boundary value problems for the viscous thin film equation:

$$
\left\{\begin{array}{l}
u_{t}-\left(m(u) w_{x}\right)_{x}=0 \text { in } Q_{T}, \\
w=-u_{x x}+v u_{t} \text { in } Q_{T}, \\
u_{x}=w_{x}=0 \text { on } \Gamma \\
u(x, 0)=u_{0}(x),
\end{array}\right.
$$

where $T>0, m(u)=u, \Omega=(-1,1), Q_{T}=\Omega \times(0, T)$ and $\Gamma=\partial \Omega \times(0, T)$.

Formally, if we substitute the second equation into the first one, we can get another form for this question:

$$
\left\{\begin{array}{l}
u_{t}+\left(m(u)\left(u_{x x}-v u_{t}\right)_{x}\right)_{x}=0 \text { in } Q_{T}, \\
u_{x}=u_{x x x}=0 \text { on } \Gamma \\
u(x, 0)=u_{0}(x)
\end{array}\right.
$$

Our main result is the following theorems.

Theorem 1. Let $u_{0} \in L^{2}(\Omega)$ and $v>0$. Then there exists at least one pair $(u, w)$ of $(1)$ satisfying

1) $u \in L^{\infty}\left(0, T ; H^{1}(\Omega)\right) \cap L^{2}\left(0, T ; H^{2}(\Omega)\right) \cap C\left([0, T] ; L^{2}(\Omega)\right)$, $w \in L^{2}\left(0, T ; H^{1}(\Omega)\right) \quad u_{t} \in L^{2}\left(Q_{T}\right)$;

2) For any test function $\phi \in L^{2}\left(0, T ; H^{1}(\Omega)\right)$, it has

$$
\begin{gathered}
\iint_{Q_{T}} u_{t} \phi \mathrm{d} x \mathrm{~d} t+\iint_{Q_{T}} u w_{x} \phi_{x} \mathrm{~d} x \mathrm{~d} t=0 \\
\iint_{Q_{T}} w \phi \mathrm{d} x \mathrm{~d} t=-\iint_{Q_{T}} u_{x x} \phi \mathrm{d} x \mathrm{~d} t+v \iint_{Q_{T}} u_{t} \phi \mathrm{d} x \mathrm{~d} t .
\end{gathered}
$$

3) $u(x, 0)=u_{0}(x)$.

Theorem 2. Let $u_{0} \in L^{2}(\Omega)$ and $v>0$. Then there exists at least one pair $(u, w)$ of (2) satisfying

1) $u \in L^{\infty}\left(0, T ; H^{1}(\Omega)\right) \cap L^{2}\left(0, T ; H^{2}(\Omega)\right) \cap C\left([0, T] ; L^{2}(\Omega)\right), u_{t} \in L^{2}\left(Q_{T}\right)$;

2) For any test function $\phi \in L^{2}\left(0, T ; H^{2}(\Omega)\right)$ with $\phi_{x}(-1, t)=\phi_{x}(1, t)=0$, it has

$$
\iint_{Q_{T}} u_{t} \phi \mathrm{d} x \mathrm{~d} t+\iint_{Q_{T}} u_{x x} u_{x} \phi_{x} \mathrm{~d} x \mathrm{~d} t-v \iint_{Q_{T}} u u_{t} \phi_{x x} \mathrm{~d} x \mathrm{~d} t=0 .
$$


3) $u(x, 0)=u_{0}(x)$.

The following lemmas are needed in the paper:

Lemma 1. (Aubin-Lions, see [13]) Let $X, B$ and $Y$ be Banach spaces and assume $X \rightarrow B \rightarrow Y$ with compact imbedding $X \rightarrow B$.

1) Let $\mathfrak{F}$ be bounded in $L^{p}(0, T ; X)$ where $1 \leq p<\infty$, and $\frac{\partial \mathfrak{F}}{\partial t}=\left\{\frac{\partial f}{\partial t}: f \in \mathfrak{F}\right\}$ be bounded in $L^{1}(0, T ; Y)$. Then $\mathfrak{F}$ is relatively compact in $L^{p}(0, T ; B)$;

2) Let $\mathfrak{F}$ be bounded in $L^{\infty}(0, T ; X)$, and $\frac{\partial \mathfrak{F}}{\partial t}=\left\{\frac{\partial f}{\partial t}: f \in \mathfrak{F}\right\}$ be bounded in $L^{r}(0, T ; Y)$ where $r>1$. Then $\mathfrak{F}$ is relatively compact in $C([0, T] ; B)$.

Lemma 2. (see [14] or [15]) Let $V$ be a real, separable, reflexive Banach space and $H$ is a real, separable, Hilbert space. $V \rightarrow H$ is continuous and $V$ is dense in $H$. Then $\left\{u \in L^{2}(0, T ; V) \mid u_{t} \in L^{2}\left(0, T ; V^{\prime}\right)\right\}$ is continuously imbedded in $C([0, T] ; H)$.

In this paper, $C$ is denoted as a positive constant and may change from line to line. The paper is arranged as follows. The existence of solutions to the approximate problem will be proved in Section 2. In Section 3, we will take the limit for small parameters $\delta \rightarrow 0$.

\section{Approximate Problem}

For any $0<\delta<1$, we consider the following approximate problem. In order to apply existence theory better, we transform (1) into a system:

$$
\left\{\begin{array}{l}
u_{\delta t}-\left(m_{\delta}\left(u_{\delta}\right) w_{\delta x}\right)_{x}=0 \text { in } Q_{T}, \\
w_{\delta}=-u_{\delta x x}+v u_{\delta t} \text { in } Q_{T}, \\
u_{\delta x}=w_{\delta x}=0 \text { on } \Gamma \\
u_{\delta}(x, 0)=u_{\delta 0}(x)
\end{array}\right.
$$

with $u_{\delta 0}(x)=u_{0}(x)+\delta, m_{\delta}\left(u_{\delta}\right)=u_{\delta+}+\delta$ and $u_{\delta+}=\max \left\{u_{\delta}, 0\right\}$.

Lemma 3. There exists at least one solution $u_{\delta}$ to (3) satisfying

1) $w_{\delta} \in L^{2}\left(0, T ; H^{1}(\Omega)\right)$,

$u_{\delta} \in L^{2}\left(0, T ; H^{2}(\Omega)\right) \cap L^{\infty}\left(0, T ; H^{1}(\Omega)\right) \cap C\left([0, T] ; L^{2}(\Omega)\right), \quad u_{\delta t} \in L^{2}\left(Q_{T}\right)$ and $u_{\delta}(x, 0)=u_{\delta 0}$;

2) For any test function $\phi \in L^{2}\left(0, T ; H^{1}(\Omega)\right)$, it has

$$
\begin{gathered}
\iint_{Q_{T}} u_{\delta t} \phi \mathrm{d} x \mathrm{~d} t+\iint_{Q_{T}} m_{\delta}\left(u_{\delta}\right) w_{\delta x} \phi_{x} \mathrm{~d} x \mathrm{~d} t=0, \\
\iint_{Q_{T}} w_{\delta} \phi \mathrm{d} x \mathrm{~d} t=-\iint_{Q_{T}} u_{\delta x x} \phi \mathrm{d} x \mathrm{~d} t+v \iint_{Q_{T}} u_{\delta t} \phi \mathrm{d} x \mathrm{~d} t .
\end{gathered}
$$

Proof. We apply the Galerkin method to prove this Lemma and so we choose $\left\{\phi_{i}\right\}_{i=1,2,3, \ldots}$ as the eigenfunctions of the Laplace operator with Neumann boundary value conditions such that $-\phi_{i x x}=\lambda_{i} \phi_{i}$. Moreover, we can suppose that the eigenfunctions are orthogonal in the $H^{1}$ and $L^{2}$ spaces. We use $(\cdot, \cdot)$ to denote the scalar product in $L^{2}$ space and we can normalize $\phi_{i}$ such that 
$\left(\phi_{i}, \phi_{j}\right)=\delta_{i j}=\left\{\begin{array}{ll}1, & i=j, \\ 0, & i \neq j .\end{array}\right.$ Besides, we can choose $\lambda_{1}=0$ and $\phi_{1}=1$.

For any positive integer $M$, we define $u_{\delta}^{M}(x, t)=\sum_{i=1}^{M} c_{i}(t) \phi_{i}(x)$,

$u_{\delta}^{M}(x, 0)=\sum_{i=1}^{M}\left(u_{0}, \phi_{i}\right) \phi_{i}, \quad w_{\delta}^{M}(x, t)=\sum_{i=1}^{M} d_{i}(t) \phi_{i}(x)$. Now we consider the following ordinary differential equations system:

$$
\begin{gathered}
\frac{\mathrm{d}}{\mathrm{d} t}\left(u_{\delta}^{M}, \phi_{j}\right)=-\left(m_{\delta}\left(u_{\delta}^{M}\right) w_{\delta x}^{M}, \phi_{j x}\right), \\
\left(w_{\delta}^{M}, \phi_{j}\right)=-\left(u_{\delta x x}^{M}, \phi_{j}\right)+v \frac{\mathrm{d}}{\mathrm{d} t}\left(u_{\delta}^{M}, \phi_{j}\right),
\end{gathered}
$$

for $j=1, \cdots, M$, which yields an initial value problem for the ordinary differential equations:

$$
\begin{gathered}
\frac{\mathrm{d}}{\mathrm{d} t} c_{j}+v \sum_{i=1}^{M} \frac{\mathrm{d}}{\mathrm{d} t} c_{i}(t)\left(m_{\delta}\left(\sum_{i=1}^{M} c_{i}(t) \phi_{i}(x)\right) \phi_{i x}, \phi_{j x}\right) \\
=-\sum_{i=1}^{M} c_{i}(t)\left(m_{\delta}\left(\sum_{i=1}^{M} c_{i}(t) \phi_{i}(x)\right) \phi_{i x}, \phi_{j x}\right), \\
c_{j}(0)=\left(u_{\delta 0}, \phi_{j}\right),
\end{gathered}
$$

with $j=1, \cdots, M$. A standard argument can show that this ODE has a local solution by Peano existence theorem since the matrix $\left(m_{\delta}\left(\sum_{i=1}^{M} c_{i}(t) \phi_{i}(x)\right) \phi_{i x}, \phi_{j x}\right)$ is positive definite. In order to get the global solvability, we need establish more energy estimates. Multiply (4) by $w_{\delta}^{M}$ to get

$$
\int_{\Omega} u_{\delta t}^{M} w_{\delta}^{M} \mathrm{~d} x=-\int_{\Omega}\left(u_{\delta+}^{M}+\delta\right)\left|w_{\delta}^{M}\right|^{2} \mathrm{~d} x .
$$

Taking $u_{\delta t}^{M}$ as the test function in (5), we have

$$
\int_{\Omega} u_{\delta t}^{M} w_{\delta}^{M} \mathrm{~d} x=-\int_{\Omega} u_{\delta x x}^{M} u_{\delta t}^{M} \mathrm{~d} x+v \int_{\Omega}\left|u_{\delta t}^{M}\right|^{2} \mathrm{~d} x .
$$

Thus, we have

$$
\frac{1}{2} \frac{\mathrm{d}}{\mathrm{d} t} \int_{\Omega}\left|u_{\delta x}^{M}\right|^{2} \mathrm{~d} x+v \int_{\Omega}\left|u_{\delta t}^{M}\right|^{2} \mathrm{~d} x+\int_{\Omega}\left(u_{\delta+}^{M}+\delta\right)\left|w_{\delta x}^{M}\right|^{2} \mathrm{~d} x=0 .
$$

Therefore, for any $0<t \leq T$, it has

$$
\begin{aligned}
& \frac{1}{2} \int_{\Omega}\left|u_{\delta x}^{M}\right|^{2}(x, t) \mathrm{d} x+v \iint_{Q_{t}}\left|u_{\delta t}^{M}\right|^{2} \mathrm{~d} x \mathrm{~d} t+\iint_{Q_{t}}\left(u_{\delta+}^{M}+\delta\right)\left|w_{\delta x}^{M}\right|^{2} \mathrm{~d} x \mathrm{~d} t \\
& \leq C \int_{\Omega}\left|u_{\delta 0 x}\right|^{2} \mathrm{~d} x .
\end{aligned}
$$

Since $\frac{\mathrm{d}}{\mathrm{d} t} \int_{\Omega} u_{\delta}^{M} \mathrm{~d} x=0$ by (4) with $j=1$, we can apply Poincaré's inequality to obtain the following estimates:

$$
u_{\delta}^{M} \in L^{\infty}\left(0, T ; H^{1}(\Omega)\right), u_{\delta t}^{M} \in L^{2}\left(Q_{T}\right), w_{\delta}^{M} \in L^{2}\left(0, T ; H^{1}(\Omega)\right) .
$$

By taking $u_{\delta x x}^{M}$ as the test function in (5), we have 


$$
\int_{\Omega} u_{\delta x x}^{M} w_{\delta}^{M} \mathrm{~d} x=-\int_{\Omega}\left|u_{\delta x x}^{M}\right|^{2} \mathrm{~d} x-\frac{1}{2} \frac{\mathrm{d}}{\mathrm{d} t} v \int_{\Omega}\left|u_{\delta x}^{M}\right|^{2} \mathrm{~d} x .
$$

By integrating over $(0, T)$ and applying the Höler's inequality, we have

$$
\begin{aligned}
& v \int_{\Omega}\left|u_{\delta x}^{M}\right|^{2}(x, T) \mathrm{d} x+\iint_{Q_{T}}\left|u_{\delta x x}^{M}\right|^{2} \mathrm{~d} x \mathrm{~d} t \\
& \leq C v \int_{\Omega}\left|u_{\delta 0 x}\right|^{2} \mathrm{~d} x+\iint_{Q_{T}}\left|w_{\delta}^{M}\right|^{2} \mathrm{~d} x \leq C .
\end{aligned}
$$

which yields

$$
u_{\delta}^{M} \in L^{2}\left(0, T ; H^{2}(\Omega)\right) .
$$

There exists a subsequence of $\left(u_{\delta}^{M}, w_{\delta}^{M}\right)$ and a pair $\left(u_{\delta}, w_{\delta}\right)$ such that, as $M \rightarrow \infty$,

$$
\begin{gathered}
u_{\delta}^{M} \rightarrow u_{\delta} \text { weakly* in } L^{\infty}\left(0, T ; H^{1}(\Omega)\right), \\
u_{\delta t}^{M} \rightarrow u_{\delta t} \text { weakly in } L^{2}\left(Q_{T}\right), \\
w_{\delta}^{M} \rightarrow w_{\delta} \text { weakly in } L^{2}\left(0, T ; H^{1}(\Omega)\right), \\
u_{\delta}^{M} \rightarrow u_{\delta} \text { weakly in } L^{2}\left(0, T ; H^{2}(\Omega)\right), \\
u_{\delta}^{M} \rightarrow u_{\delta} \text { strongly in } C\left([0, T] ; L^{2}(\Omega)\right) \text { and a.e. in } Q_{T} .
\end{gathered}
$$

where the last estimate is from Lemma 1. By (13)-(17), we can perform the limit $M \rightarrow \infty$ in a standard fashion and the strong convergence in $C\left([0, T] ; L^{2}(\Omega)\right)$ implies $u_{\delta}(x, 0)=u_{\delta 0}(x)$.

\section{The Limit $\delta \rightarrow 0$}

In the section, we will perform the limit $\delta \rightarrow 0$ to the solutions from Lemma 3 . For the purpose of the existence, we need establish some uniform estimates independent of $\delta$. Thus, we define a convex function $\Phi_{\delta}(\cdot)$ as following (see [10]):

$$
\Phi_{\delta}(\sigma)= \begin{cases}(\sigma+\delta) \ln (\sigma+\delta)-(\sigma+\delta)+1, & \sigma \geq 0 \\ \frac{(\sigma)^{2}}{2 \delta}+\sigma(\ln \delta)+\delta(\ln \delta)-\delta+1, & \sigma<0\end{cases}
$$

Moreover, the function $\Phi_{\delta}$ satisfies $\Phi_{\delta} \geq 0, \Phi_{\delta} \in W_{l o c}^{2,+\infty}(R)$, $\Phi_{\delta}^{\prime \prime}(\sigma)=\frac{1}{\sigma_{+}+\delta}$.

By applying this function, we can get the following estimates.

Lemma 4. There exist some constants $C$ independent of $\delta$ such that
1) $\left\|u_{\delta}\right\|_{L^{\infty}\left(0, T ; H^{1}(\Omega)\right)} \leq C$;
2) $\left\|u_{\delta}\right\|_{L^{\infty}\left(Q_{T}\right)} \leq C$;
3) $\left\|u_{\delta}\right\|_{L^{2}\left(0, T ; H^{2}(\Omega)\right)} \leq C$;
4) $\iint_{Q_{T}}\left(u_{\delta+}+\delta\right)\left|w_{\delta}\right|^{2} \mathrm{~d} x \mathrm{~d} t \leq C$;
5) $\left\|u_{\delta t}\right\|_{L^{2}\left(Q_{T}\right)} \leq C$; 
6) $\left\|w_{\delta}\right\|_{L^{2}\left(Q_{T}\right)} \leq C$.

Proof. Taking $\Phi^{\prime}\left(u_{\delta}\right)$ as a test function in the first equation of (3), we have

$$
\frac{\mathrm{d}}{\mathrm{d} t} \int_{\Omega}\left(\Phi\left(u_{\delta}(x, t)\right)+\frac{v}{2} u_{\delta x}^{2}\right) \mathrm{d} x+\int_{\Omega}\left|u_{\delta x x}\right|^{2} \mathrm{~d} x=0
$$

Thus, it yields the results $1-3$. We can prove 4 and 5 from (8). By choosing $w_{\delta}$ as a test function in the second equation of (3), we get

$$
\begin{aligned}
\iint_{Q_{T}} w_{\delta}^{2} \mathrm{~d} x \mathrm{~d} t & \leq \frac{1}{2} \iint_{Q_{T}} w_{\delta}^{2} \mathrm{~d} x \mathrm{~d} t+C \iint_{Q_{T}} u_{\delta x x} \mathrm{~d} x \mathrm{~d} t+C \iint_{Q_{T}}\left|u_{\delta t}\right|^{2} \mathrm{~d} x \mathrm{~d} t \\
& \leq \frac{1}{2} \iint_{Q_{T}} w_{\delta}^{2} \mathrm{~d} x \mathrm{~d} t+C .
\end{aligned}
$$

We have completed the proof of this lemma.

Lemma 5. There exists a pair $(u, w)$ such that, as $\delta \rightarrow 0$,

1) $u_{\delta} \rightarrow u$ weakly* in $L^{\infty}\left(0, T ; H^{1}(\Omega)\right)$;

2) $u_{\delta t} \rightarrow u_{t}$ weakly in $L^{2}\left(Q_{T}\right)$;

3) $u_{\delta} \rightarrow u$ strongly in $C\left([0, T] ; L^{2}(\Omega)\right)$ and a.e. in $Q_{T}$;

4) $w_{\delta} \rightarrow w$ weakly in $L^{2}\left(Q_{T}\right)$;

5) $u \geq 0$ a.e. in $Q_{T}$.

Proof. By Lemma 4, we can get the results 1 - 2 and 4 directly. Lemma 1 yields 3. By applying the definition of $\Phi_{\delta}(\cdot)$ and (18), we get

$$
0 \leq \frac{1}{2} \int_{\Omega} u_{\delta-}^{2}(x, t) \mathrm{d} x \leq \delta \int_{\Omega} \Phi_{\delta}\left(u_{\delta}\right) \mathrm{d} x-\delta \ln \delta \int_{\Omega} u_{\delta-}(x, t) \mathrm{d} x+C \delta .
$$

It yields

$$
0 \leq-\ln \delta \int_{\Omega} u_{\delta-}(x, t) \mathrm{d} x+C .
$$

Letting $\delta \rightarrow 0$, we obtain $\int_{\Omega} u_{-}^{2}(x, t) \mathrm{d} x=0$ which completes the proof of 6 .

Proof of Theorem 1 and Theorem 2. Taking $\phi \in L^{2}\left(0, T ; H_{0}^{2}(\Omega)\right)$ as a test function in Lemma 3, we have

$$
\begin{gathered}
\iint_{Q_{T}} u t \phi \mathrm{d} x \mathrm{~d} t+\iint_{Q_{T}} u w_{x} \phi_{x} \mathrm{~d} x \mathrm{~d} t=0, \\
\iint_{Q_{T}} w \phi \mathrm{d} x \mathrm{~d} t=-\iint_{Q_{T}} u_{x x} \phi \mathrm{d} x \mathrm{~d} t+v \iint_{Q_{T}} u_{t} \phi \mathrm{d} x \mathrm{~d} t,
\end{gathered}
$$

which yields Theorem 1 .

On the other hand, by integrating by parts, it implies

$$
\iint_{Q_{T}} u_{t} \phi \mathrm{d} x \mathrm{~d} t-\iint_{Q_{T}} w u_{x} \phi_{x} \mathrm{~d} x \mathrm{~d} t-v \iint_{Q_{T}} u w \phi_{x x} \mathrm{~d} x \mathrm{~d} t=0 .
$$

Thus, it has

$$
\begin{aligned}
& \iint_{Q_{T}} u_{t} \varphi \mathrm{d} x \mathrm{~d} t+\iint_{Q_{T}} u_{x x} u_{x} \phi_{x} \mathrm{~d} x \mathrm{~d} t+\iint_{Q_{T}} u u_{x x} \phi_{x x} \mathrm{~d} x \mathrm{~d} t \\
& -v \iint_{Q_{T}} u_{x} u_{t} \phi_{x} \mathrm{~d} x \mathrm{~d} t-v \iint_{Q_{T}} u u_{t} \phi_{x x} \mathrm{~d} x \mathrm{~d} t=0 .
\end{aligned}
$$

It gives Theorem 2 .

\section{Conclusions}

Through this paper, two forms of a viscous thin film equation are studied (see 
the Equations (1) and (2)) and we give the corresponding existence theorems of weak solutions (see Theorem 1 and Theorem 2). For any test function $\phi$, we have proved that the weak solutions satisfy the equalities:

$$
\begin{gathered}
\iint_{Q_{T}} u_{t} \phi \mathrm{d} x \mathrm{~d} t+\iint_{Q_{T}} u w_{x} \phi_{x} \mathrm{~d} x \mathrm{~d} t=0, \\
\iint_{Q_{T}} w \phi \mathrm{d} x \mathrm{~d} t=-\iint_{Q_{T}} u_{x x} \phi \mathrm{d} x \mathrm{~d} t+v \iint_{Q_{T}} u_{t} \phi \mathrm{d} x \mathrm{~d} t .
\end{gathered}
$$

Since the thin film equation is a degenerate parabolic equation, it is hard to give the existence of strong solutions. On the another hand, the viscous term affects the regularity of solutions and we have shown that $u_{t} \in L^{2}\left(Q_{T}\right)$.

We can expect that we can show that the existence results would be true with some conditions in high-dimensional space.

\section{Funding}

The work was supported by the Education Department Science Foundation of Liaoning Province of China (No. JDL2016029) and the Natural Science Fund of Liaoning Province of China (No. 20170540136).

\section{Conflicts of Interest}

The authors declare that they have no competing interests.

\section{References}

[1] Cahn, J.M. and Hilliard, J.E. (1958) Free Energy of a Non-Uniform System I. Interfacial Free Energy. The Journal of Chemical Physics, 28, 258-367. https://doi.org/10.1063/1.1744102

[2] Elliott, C.M. and Zheng, S. (1986) On the Cahn Hilliard Equation. Archive for Rational Mechanics and Analysis, 96, 339-357. https://doi.org/10.1007/BF00251803

[3] Elliott, C.M. and Garcke, H. (1996) On the Cahn Hilliard Equation with Degenerate Mobility. SIAM Journal on Mathematical Analysis, 27, 404-423. https://doi.org/10.1137/S0036141094267662

[4] Xu, M. and Zhou, S. (2005) Existence and Uniqueness of Weak Solutions for a Generalized Thin Film Equation. Nonlinear Analysis. Theory, Methods \& Applications, 60, 755-774. https://doi.org/10.1016/j.na.2004.01.013

[5] Xu, M. and Zhou, S. (2008) Stability and Regularity of Weak Solutions for a Generalized Thin Film Equation. Journal of Mathematical Analysis and Applications, 337, 49-60. https://doi.org/10.1016/j.jmaa.2007.03.075

[6] Liang, B. and Zheng, S. (2008) Existence and Asymptotic Behavior of Solutions to a Nonlinear Parabolic Equation of Fourth Order. Journal of Mathematical Analysis and Applications, 348, 234-243. https://doi.org/10.1016/j.jmaa.2008.07.022

[7] Myers, T.G. (1998) Thin Films with High Surface Tension. SIAM Reviews, 40, 441-462. https://doi.org/10.1137/S003614459529284X

[8] Bernis, F. and Friedman, A. (1990) Higher Order Nonlinear Degenerate Parabolic Equations. Journal of Differential Equations, 83, 179-206. https://doi.org/10.1016/0022-0396(90)90074-Y

[9] Bertozzi, A.L. and Pugh, M. (1994) The Lubrication Approximation for Thin Viscous Films. Nonlinearity, 7, 1535-1564. https://doi.org/10.1088/0951-7715/7/6/002 
[10] Boutat, M., Hilout, S., Rakotoson, J.E., et al. (2008) A Generalized Thin-Film Equation in Multidimensional Space. Nonlinear Analysis, 69, 1268-1286.

https://doi.org/10.1016/j.na.2007.06.028

[11] Ansini, L. and Giacomelli, L. (2004) Doubly Nonlinear Thin-Film Equations in One Space Dimension. Archive for Rational Mechanics and Analysis, 173, 89-131. https://doi.org/10.1007/s00205-004-0313-x

[12] Beretta, E., Bertsch, M. and Dal Passo, R. (1995) Nonnegative Solutions of a Fourth-Order Nonlinear Degenerate Parabolic Equation. Archive for Rational Mechanics and Analysis, 129, 175-200. https://doi.org/10.1007/BF00379920

[13] Simon, J. (1987) Compact Sets in the Space $L^{p}(0, T ; B)$. Annali di Matematica Pura ed Applicata, 146, 65-96. https://doi.org/10.1007/BF01762360

[14] Grün, G. (1995) Degenerate Parabolic Differential Equations of Fourth Order and a Plasticity Model with Nonlocal Hardening. Zeitschrift für Analysis und Ihre Anwendungen, 14, 541-574. https://doi.org/10.4171/ZAA/639

[15] Zeidler, E. (1997) Nonlinear Functional Analysis and Its Applications. Springer, New York. 